\title{
Spendebereitschaft basiert auf Vertrauen
}

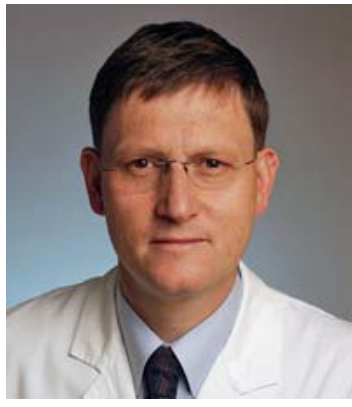

H. Klüter

\author{
Verehrte Leser, liebe Kolleginnen \\ und Kollegen, \\ $\nabla$
}

die anhaltende Diskussion um die Allokation von Spenderorganen zur Organtransplantation macht auch die in der Transfusionsmedizin tätigen und mit der Blutübertragung betrauten Kolleginnen und Kollegen betroffen. Einerseits sind viele transfusionsmedizinische Einrichtungen in die Gewebetypisierungen bei Transplantationen eingebunden. Mit ihrer Kompetenz stehen sie ein für die bestmögliche Kompatibilität zwischen einem Spenderorgan und dem Organempfänger und sichern somit die immungenetische Rationale der Organverteilung. Die letzte Ausgabe (Heft 4/2012) hat sich diesem Thema gewidmet.

Andererseits stellt die allogene Blutübertragung eine besondere Form der Transplantation dar. Während die ungelösten Probleme bei der Organtransplantation vor allem auf einer mangelnden Organspendebereitschaft beruhen, hat die Blutspende in Deutschland eine jahrzehntelange gesellschaftliche Tradition. Millionen von Mitbürgern vertrauen sich regelmäßig den Blutspendeeinrichtungen an und leisten mit ihrer Blutspende einen für das Gesundheitswesen unersetzbaren Beitrag. Für die Kliniken und ihre Patienten stehen dadurch "rund-um-die-Uhr“ und „rundum's-Jahr“" hochwertige Blutpräparate bereit. Diese nahezu selbstverständlich anmutende Verfügbarkeit von Blutpräparaten macht manchmal vergessen, dass hinter jeder Blutübertragung die aktive und persönliche Spendebereitschaft eines einzelnen Mitglieds unserer Gesellschaft steht.

Gerade dieses Vertrauen in die Transfusionsmedizin bedarf einer besonderen Pflege. Durch die fortlaufende Entwicklung und Etablierung von Methoden zur Untersuchung des gespendeten Blutes wurde dem Auftrag der Sicherheit bei der Blutübertragung, zuletzt besonders im Hinblick auf die Vermeidung möglicher Infektionen, mit großem Erfolg Rechnung getragen. Zur Sicherheit der Therapie mit Blutpräparaten gehört aber auch die Sicherung einer ausreichend großen Zahl an Spendewilligen. Das lehrt uns das Beispiel aus der Transplantationsmedizin!

Dabei kommt der rationalen Indikation zur Blutübertragung eine herausragende Bedeutung zu. Die altruistische Blutspende basiert auf dem Vertrauen, mit einer Blutspende konkret helfen zu können. Klare Empfehlungen für die Anwendung von Blutpräparaten sichern den klinischen Einsatz dieser begrenzten Ressource und beugen einer missbräuchlichen Verwendung vor. In dieser Ausgabe widmet sich der CME-Beitrag von
Janetzko, Ebell und Welte der Transfusion von Erythrozytenkonzentraten. Deren Einsatz in klinischen Situationen bedingt eine differenzielle Therapieentscheidung. Der benutzte Begriff des „restriktiven Einsatzes von Erythrozytenkonzentraten“ lässt erkennen, dass die aus der aktuellen Literatur und den nationalen und internationalen Leitlinien zusammengetragenen Empfehlungen dem Primat einer Schonung der Ressource Blut folgen, ohne dass dabei Abstriche bei der Sicherstellung des Erfolgs der Behandlung akzeptiert werden.

Die Beachtung dieser aktuellen Empfehlungen sichert somit eine auch zukünftig unersetzbare Bereitschaft der Blutspender zur Mitwirkung. Keinesfalls darf diese Spendebereitschaft durch unreflektierte populistische Äußerungen im Hinblick auf eine vermeintlich nicht indizierte Verwendung von Blutpräparaten gefährdet werden. Ein offener Wissens- und Erfahrungsaustausch zwischen Ärztinnen und Ärzten, wie es die Internet-basierte Plattform „Klinische Hämotherapie“ möglich macht, ist deshalb wichtig. E.K. Petershofen stellt diese Initiative für die Fort- und Weiterbildung in der Rubrik „Praxistipp“ nochmals vor.

Viel hat auch das Transfusionsgesetz (TFG) zu dem hohen gesellschaftlichen Vertrauen im Umgang mit Blutpräparaten beigetragen. Es besteht nunmehr 15 Jahre. Der ehemals im Gesundheitsministerium verantwortlich tätige Friedger von Auer geht in seinem Beitrag auf diesen Jahrestag ein. Das Transfusionsgesetz legt die Aufgaben der im Blutspendewesen und in der klinischen Hämotherapie handelnden Institutionen (PEI, BÄK, RKI), Blutspendeeinrichtungen, Fachgesellschaften, Transfusionsmediziner und Ärzte anderer Berufsgruppen sowie der Patientenverbände fest. Die heute erzielte Sicherheit in der Blutversorgung geht auch auf eine strukturierte Zusammenarbeit zwischen diesen Partnern zurück.

Nur eingebunden in einer konsensorientierten Partnerschaft aller im Blutspendewesen beteiligten Parteien und durch einen rationalen klinischen Umgang mit der limitierten Ressource Blut kann der für die Versorgung unverzichtbare Gesellschaftsvertrag zwischen Blutspender und Empfänger halten!

Auch im Namen der Mitherausgeber danke ich allen Autorinnen und Autoren für ihren Beitrag und Ihnen, liebe Leser, für Ihr fortwährendes Interesse an der „Transfusionsmedizin“.

Prof. Dr. med. Harald Klüter, Mannheim

Februar 2013 